Review

\title{
Improving the Magnetic Resonance Imaging Contrast and Detection Methods with Engineered Magnetic Nanoparticles
}

\author{
Jing Huang ${ }^{1,2}$, Xiaodong Zhong ${ }^{3}$, Liya Wang ${ }^{1,2}$, Lily Yang ${ }^{4}$ and Hui Mao ${ }^{1,2} \bowtie$ \\ 1. Department of Radiology and Imaging Sciences, Emory University School of Medicine, Atlanta, Georgia 30322, USA \\ 2. Center for Systems Imaging, Emory University School of Medicine, Atlanta, Georgia 30322, USA \\ 3. MR Research and Development, Siemens Healthcare, USA \\ 4. Department of Surgery, Emory University School of Medicine, Georgia 30322, USA
}

$\triangle$ Corresponding author: Hui Mao, PhD, Department of Radiology and Imaging Sciences, Emory University School of Medicine, 1364 Clifton Road, Atlanta, Georgia 30322, USA. Phone: (404) 712-0357. Fax: (404) 712-5948. E-mail: hmao@emory.edu

(C) Ivyspring International Publisher. This is an open-access article distributed under the terms of the Creative Commons License (http://creativecommons.org/ licenses/by-nc-nd/3.0/). Reproduction is permitted for personal, noncommercial use, provided that the article is in whole, unmodified, and properly cited.

Received: 2011.11.23; Accepted: 2012.01.05; Published: 2012.01.10

\begin{abstract}
Engineering and functionalizing magnetic nanoparticles have been an area of the extensive research and development in the biomedical and nanomedicine fields. Because their biocompatibility and toxicity are well investigated and better understood, magnetic nanoparticles, especially iron oxide nanoparticles, are better suited materials as contrast agents for magnetic resonance imaging (MRI) and for image-directed delivery of therapeutics. Given tunable magnetic properties and various surface chemistries from the coating materials, most applications of engineered magnetic nanoparticles take advantages of their superb MRI contrast enhancing capability as well as surface functionalities. It has been found that MRI contrast enhancement by magnetic nanoparticles is highly dependent on the composition, size and surface properties as well as the degree of aggregation of the nanoparticles. Therefore, understanding the relationships between these intrinsic parameters and the relaxivities that contribute to MRI contrast can lead to establishing essential guidance that may direct the design of engineered magnetic nanoparticles for theranostics applications. On the other hand, new contrast mechanism and imaging strategy can be developed based on the novel properties of engineered magnetic nanoparticles. This review will focus on discussing the recent findings on some chemical and physical properties of engineered magnetic nanoparticles affecting the relaxivities as well as the impact on MRI contrast. Furthermore, MRI methods for imaging magnetic nanoparticles including several newly developed MRI approaches aiming at improving the detection and quantification of the engineered magnetic nanoparticles are described.
\end{abstract}

Key words: magnetic nanoparticles, engineering, functionalizing, magnetic resonance imaging

\section{Introduction}

In recent decades engineered magnetic nanoparticles and related nanoconstructs have attracted extensive research and development in the field of nanomedicine.[1-8] Because their biocompatibility and toxicity are extensively investigated and better un- derstood, magnetic nanoparticles, especially iron oxide nanoparticles (IONPs) composed of maghemite or magnetite nanocrystals, are proper choices for various in vivo biomedical applications. Among them, magnetic resonance imaging (MRI) contrast enhancement 
for molecular imaging takes advantage of superb and tunable magnetic properties of engineered magnetic nanoparticles, while a range of surface chemistry offered by nanoparticles provides multifunctional capabilities for image-directed drug delivery. In parallel with the fast growing research in nanotechnology and nanomedicine, the continuous advance of MRI technology and the rapid expansion of MRI applications in the clinical environment further promote the research in this area.

It is well known that magnetic nanoparticles, distributed in a magnetic field, create extremely large microscopic field gradients. These microscopic field gradients cause substantial diphase and shortening of longitudinal relaxation time $\left(\mathrm{T}_{1}\right)$ and transverse relaxation time $\left(\mathrm{T}_{2}\right.$ and $\left.\mathrm{T}_{2}{ }^{*}\right)$ of nearby nuclei, e.g., proton in the case of most MRI applications.[9, 10] The magnitudes of MRI contrast enhancement over clinically approved conventional gadolinium chelate contrast agents combined with functionalities of biomarker specific targeting enable the early detection of diseases at the molecular and cellular levels with engineered magnetic nanoparticles.[4, 6, 11, 12] While the effort in developing new engineered magnetic nanoparticles and constructs with new chemistry, synthesis, and functionalization approaches continues to grow, the importance of specific material designs and proper selection of imaging methods have been increasingly recognized. Earlier investigations have shown that the MRI contrast enhancement by magnetic nanoparticles is highly related to their composition, size, surface properties, and the degree of aggregation in the biological environment.[13-15] Therefore, understanding the relationships between these intrinsic parameters and relaxivities of nuclei under influence of magnetic nanoparticles can provide critical information for predicting the properties of engineered magnetic nanoparticles and enhancing their performance in the MRI based theranostic applications. On the other hand, new contrast mechanisms and imaging strategies can be applied based on the novel properties of engineered magnetic nanoparticles. The most common MRI sequences, such as the spin echo (SE) or fast spin echo (FSE) imaging and gradient echo (GRE), have been widely used for imaging of magnetic nanoparticles due to their common availabilities on commercial MRI scanners. In order to minimize the artificial effect of contrast agents and provide a promising tool to quantify the amount of imaging probe and drug delivery vehicles in specific sites, some special MRI methods, such as off-resonance saturation (ORS) imaging and ultrashort echo time (UTE) imaging, have been developed recently to take maximum advantage of engineered magnetic nanoparticles.[16-19]

This review will focus on discussing recent progresses made in understanding intrinsic factors that affect the relaxivity of engineered magnetic nanoparticles as well as the impact on MRI contrast. Furthermore, emerging new MRI techniques specifically developed for utilizations of magnetic nanoparticles as imaging probes and imaging detectable drug delivery carriers are described with a special focus on imaging of magnetic iron oxide nanoparticles (IONPs).

\section{Engineered Magnetic Nanoparticles for Targeted Molecular Imaging by MRI and Image-Guided Delivery of Therapeutics}

Several classes of magnetic iron oxide nanoparticles, also known as superparamagnetic IONPs (SPIO) and ultrasmall SPIO (USPIO) developed in 1980s, has been approved by FDA (e.g., Feridex) for clinical applications with capabilities of traditional "blood pool" agents.[20, 21] The important properties of cell phagocytosis of magnetic nanoparticles has expanded the applications of contrast enhanced MRI beyond the vascular and tissue morphology imaging, enabling many novel applications of magnetic IONPs for MRI diagnosis of liver diseases, cancer metastasis to lymph nodes, and in vivo tracking of implanted cell and grafts with MRI.[21-26] However, the specificities of these dextran coated magnetic nanoparticles in disease diagnosis are limited. The magnitude of contrast effects also need to be improved for high sensitivity to the minimal changes in the disease and for biomarker specific detection. Therefore, the functionalized and engineered magnetic nanoparticles are developed to meet the increasing interests for non-invasive in vivo imaging of molecular and cellular activities that target a disease.

To gain the specificity and reduce the side effect and toxicity, biomarker targeted functional proteins or peptide fragments, such as RGD targeting $\alpha_{v} \beta_{3}$ integrin, HER2/neu antibody, urokinase type plasminogen activator (uPA) amino-terminal fragment (ATF), and single chain anti-epidermal growth factor receptor (EGFR) antibody were conjugated on the surface of magnetic nanoparticles, rendering the nanoprobes being recognized and internalized by tumor cells over expressing the specific receptor.[15, 27-30] For example, Yang and co-workers developed a novel cell surface receptor-targeted MRI nanoprobe by using a recombinant peptide ATF of uPA conjugated to magnetic iron oxide (ATF-IO) nanoparticles to target uPA receptor (uPAR).[27] 


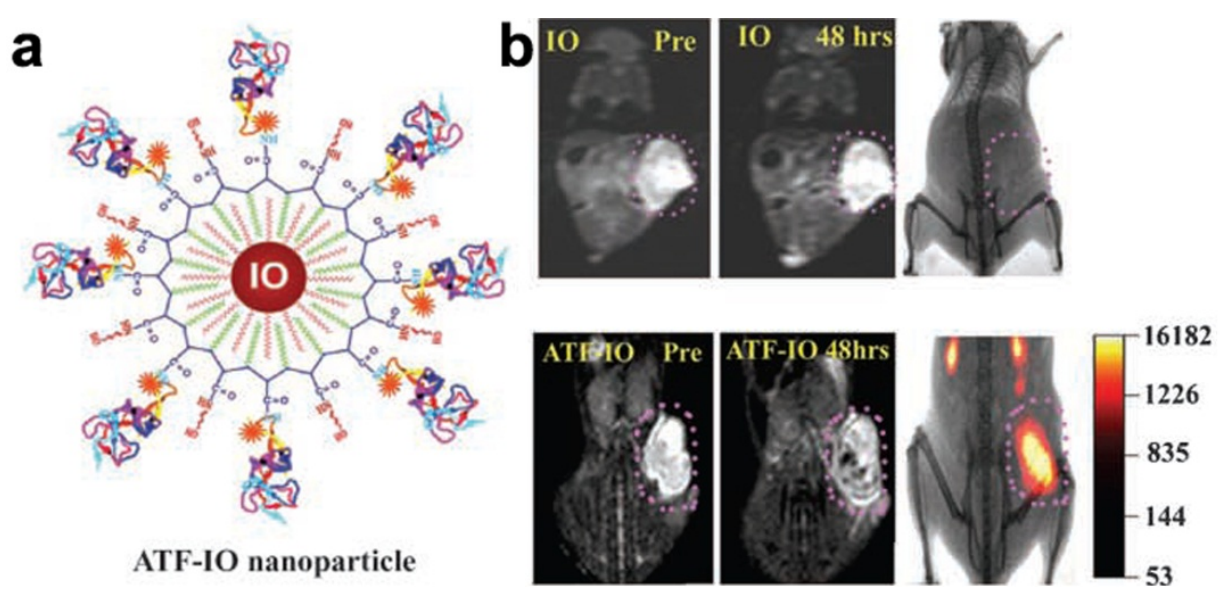

Figure I. (a) Illustration of Cy5.5-ATF-IO nanoparticles; (b) dual modality imaging ( $\mathrm{T}_{2}$-weighted MRI (left two) and NIRF imaging (right)) of subcutaneous 4TI mouse mammary tumor using Cy5.5-ATF-IO nanoparticles (bottom), no contrast change or optical signal is detected in the mouse that received nontargeted iron oxide nanoparticles (top). Adapted with permission from the reference [27].

ATF-IO nanoparticles can bind specifically to breast cancer cells expressing UPAR followed by cellular internalization through receptor mediated endocytosis. This leads to prolonged and enhanced MRI contrast in subcutaneous and intraperitoneal mammary tumors in $T_{2}$ weighted MRI (Figure 1) after intravenous administration of ATF-IO nanoparticles into tumor bearing mice. Furthermore, the receptor targeted MRI can be confirmed by optical by near-infrared fluorescence (NIRF) fluorescence imaging in mouse tumor models by co-labeling ATF-IO nanoparticles with the NIRF dye molecule, Cy5.5. This study and the developed UPAR-targeted ATF-IO nanoparticles demonstrated capabilities of functionalized magnetic nanoparticles and showed a potential to improve the specificity of the detection of human cancer by receptor targeted molecular MRI with magnetic nanoparticle probes. More importantly, researchers recognized the importance of the size effect on the relaxivity of the magnetic nanoparticle based MRI contrast. Unlike the earlier studies that used size variable SPIO made mostly with co-precipitation methods in the aqueous medium, ATF-IONPs developed in this study were consisted of size uniformed IONPs (i.e., core size of $10 \mathrm{~nm}$ measured by TEM) engineered by using the heat-deposition approach with hydrophobic medium.

Because one of the major limitations of MRI is its relative low sensitivity, the strategies of combining MRI with other highly sensitive, but less anatomically informative imaging modalities such as positron emission tomography (PET) and NIRF imaging, are extensively investigated. The complementary strengths from different imaging methods can be re- alized by using engineered magnetic nanoparticles via surface modifications and functionalizations. In order to combine optical or nuclear with MR for multimodal imaging, optical dyes and radio-isotope labeled tracer molecules are conjugated onto the moiety of magnetic nanoparticles.[31-33] Xie et al. demonstrated the multifunctionalizations of magnetic nanoparticles via dopamine-human serum albumin (HSA) procedure.[32] After exchanging the existing oleic acid/oleic amine, the dopamine modified magnetic nanoparticles can be easily encapsulated in HSA molecules for further functionalizing. In addition to MRI contrast enhancement from the core of the iron oxide nanoparticle, the covalent binding and absorption of ${ }^{64} \mathrm{Cu}$-DOTA and Cy5.5 provide PET/NIRF imaging capabilities. The application of multimodality MRI/PET/NIRF imaging using this novel probe was demonstrated in the U87MG glioma tumor model bearing mice. Due to the prolonged circulation time, the multifunctional HSA-IO nanoparticles specifically accumulated in the tumor site through passively targeting based on the tumor growth associated enhanced permeability and retention (EPR) effect. Histological examinations and analyses showed that the nanoconstructs were distributed intra-vascularly at the tumor section and not related to the uptake of macrophages.

As an expansion from the molecular imaging for MRI based diagnosis, engineered magnetic nanoparticles have been also developed for the MRI-guided delivery of therapeutic agents. For example chemotherapeutic drug noscapine (Nos) was attached on the human ATF (hATF) peptide conjugated IONPs to target UPAR expressed prostate cancer, so that the 
MRI contrast generated from IONPs can be used to follow Nos-hATF-IO nanoparticles for MRI-directed prostate cancer therapy.[34] The drug carrying uPAR targeting magnetic nanoparticles showed specific binding to UPAR expressing PC-3 cells. While these Nos-hATF-IO nanoparticles with drug molecules embedded in the hydrophobic coating layers of IONPs demonstrated a $\sim 6$-fold improvement in drug efficacy, compared to free drug, they also retained the MRI contrast effect from the IONPs cores. Besides small drug molecules, some specific antibodies or small interfering RNA (siRNA) which can inhibit the tumor growth have been also conjugated onto the magnetic nanoparticles for MRI-guided therapies. Hadjipanayis et al. crosslinked EGFRvIII antibodies to IONPs functionalized with carboxyl groups through the EDC/NHS reaction.[35] Significant decrease in glioblastoma cells survival was observed after the treatment by EGFRvIII-IONPs. MRI-guide convection-enhanced delivery of EGFRvIII-IONPs increased the survival of animals bearing glioblastoma xenografts.

Since most functionalities assembled by magnetic nanoparticles are accomplished by the surface modifications, the chemical and physical properties of nanoparticle surface as well as surface coating materials have considerable effects on the function and ability of MRI contrast enhancement of the nanoparticle core. The alterations of MRI contrast due to the surface modifications and functionalization as well as novel features of coating materials may play roles in applications of MRI methods in detecting and quantitatively monitor nanodrug bioavailability in the tumor tissues in vivo.

\section{Formulation of Engineered Magnetic Na- noparticles for High Relaxivities and MRI Contrast}

The longitudinal and transverse relaxivities, $R_{i}$ $(i=1,2)$, defined as the relaxation rate per unit concentration (e.g., millimole per liter) of magnetic ions, reflects the efficiency of contrast enhancement by the magnetic nanoparticles as MRI contrast agents. In general, the relaxivities are determined, but not limited, by three key aspects of the magnetic nanoparticles: (i) chemical composition, (ii) size of the particle or construct and the degree of their aggregation, and (iii) surface properties that can be manipulated by the modification and functionalization. It is also recognized that the shape of the nanoparticles can affect the relaxivities and contrast enhancement. However these shaped particles typically have increased sizes, which may limit their in vivo applications. Nevertheless, these novel magnetic nanomaterials are increasingly attractive and currently under investigation for their applications in MRI and image-directed drug delivery.

According to Koening-Keller model, the longitudinal or transverse relaxivity $R_{i}$ can be expressed as following:[36]

$$
R_{i}=\frac{1}{T_{i}^{m}}=\frac{a}{d_{N P} D} \gamma^{2} \mu^{2} C_{N P} J\left(\omega, \tau_{D}\right) \quad(i=1,2)
$$

Where $a$ is a constant, $d_{\mathrm{NP}}$ is the diameter of the nanoparticle, $D$ is the diffusion coefficient of water, $\mu$ is the magnetic moment of the nanoparticles, $\gamma$ is the gyromagnetic ratio of the water proton, $C_{N P}$ is the concentration of the nanoparticles, and $J\left(\omega, \tau_{D}\right)$ is the spectral density function. As the longitudinal (spin-lattice) relaxation $\mathrm{T}_{1}$ contrast agents affect the relaxation of water proton through direct energy exchange between water and paramagnetic ions, the magnetic moment $\mu$ is directly related to the unpaired electrons. While for the $T_{2}$ contrast agents in form of nanocrystals, the magnetic moment $\mu$ is responsible on the unpaired electrons as well as the electron interactions in the crystal structure.

\section{I Composition Effect on MRI Contrast}

The composition of magnetic nanoparticles can significantly affect the contrast enhancing capability of nanoparticles because it dominates the magnetic moment at the atomic level. For instance, the magnetic moments of the iron oxide nanoparticles, mostly used nanoparticulate $\mathrm{T}_{2}$ weighted MRI contrast agents, can be changed by incorporating other metal ions into the iron oxide. Cheon and coworkers have demonstrated

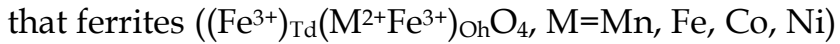
with different cations doped at the octahedral $(\mathrm{Oh})$ sites exhibit different relaxivities according to their different mass magnetization. The $\mathrm{MnFe}_{2} \mathrm{O}_{4}$ nanoparticles have the highest magnetic moment per unit allowing them to exhibit the highest relaxivity at 358 $\mathrm{mM}^{-1} \mathrm{~s}^{-1}$ at $1.5 \mathrm{~T}$ among these ferrites.[8] With $\mathrm{Zn}^{2+}$ dopants in tetrahedral (Td) sites leads to even higher magnetization value, resulting in $\mathrm{R}_{2}$ relaxivity in value of $860 \mathrm{mM}^{-1} \mathrm{~s}^{-1}$ at 4.7 T.[37] Other efforts on investigating the compositions of contrast agents were made specifically for shortening the longitudinal $\mathrm{T}_{1}$ relaxation time (e.g. $\mathrm{Gd}_{2} \mathrm{O}_{3}, \mathrm{GdF}_{3}, \mathrm{MnO}$ ). The selections of these metals are mainly based on $\mathrm{Gd}^{3+}$ or $\mathrm{Mn}^{2+}$ due to their large number of unpaired electrons. This is believed to facilitate the relaxation of protons through the interaction between the magnetic ions on the surface of the particles and the water protons in the media. Comparing to iron oxide, $\mathrm{Gd}^{3+}$ or $\mathrm{Mn}^{2+}$-based nanoparticles have relatively stronger 
paramagnetic property (large $\mathrm{R}_{1}$, i.e., $1 / \mathrm{T}_{1}$ ) and negligible magnetic anisotropy (small $\mathrm{R}_{2}$ ). For example, the $\mathrm{R}_{2} / \mathrm{R}_{1}$ value for PEG-Gd $\mathrm{d}_{2} \mathrm{O}_{3}$ contrast agents is around 1.0 while the $R_{2} / R_{1}$ value of commercial negative contrast agents, iron oxide nanoparticles such as Feridex, is around 10.0.[14, 38] Besides metal oxides, metal, and metal alloy nanoparticles are tentatively considered as novel particulate contrast agents. This is due to their extreme high saturation magnetism compared with oxides although their stability and potential toxicity in biomedical applications need to be addressed. Metallic Fe nanoparticles prepared by Hacliipanayis et al. have superb magnetism compared to iron oxide nanoparticles. The characterization of the MRI contrast effect suggested that the pure Fe nanoparticles are powerful contrast agents because of the much higher relaxivities $\left(129 \mathrm{mM}^{-1} \mathrm{~s}^{-1}\right)$ of pure Fe nanoparticles that were measured at $1.5 \mathrm{~T}$, compared to IONPs with a comparable size.[39] Dai and co-workers have reported metallic alloy nanoparticles for MRI contrast enhancement. They synthesized $\mathrm{FeCo} /$ single-graphitic-shell nanocrystals and tested their magnetic properties as MR contrast agents. High relaxivity of FeCo nanoparticles was detected at $1.5 \mathrm{~T}$ $\left(\mathrm{R}_{1}=70 \mathrm{mM}^{-1} \mathrm{~s}^{-1}, \mathrm{R}_{2}=644 \mathrm{mM}^{-1} \mathrm{~s}^{-1}\right.$ for $7 \mathrm{~nm}$ FeCo nanoparticles), and FeCo has high Ms (215 emu g-1).[38] FePt nanoparticles also exhibit high $\mathrm{R}_{2}$ relaxivity $\left(122.6 \mathrm{mM}^{-1} \mathrm{~s}^{-1}\right.$ for $4 \mathrm{~nm} \mathrm{FePt}$ nanoparticles at $0.5 \mathrm{~T}$ ) which are superior to $T_{2}$ contrast agents.[40]

\subsection{Size Effect on MRI Contrast}

The dependence of relaxation rates on the particle size has been widely studied both theoretically and experimentally. Generally the accelerated diphase, often described by the $\mathrm{R}_{2}{ }^{*}$ in magnetically inhomogeneous environment induced by magnetic nanoparticles, is predicted into two different regimes. For the relatively small nanoparticles, proton diffusion between particles is much faster than the resonance frequency shift. This resulted in the relative independence of $T_{2}$ on echo time. The values for $R_{2}$ and $R_{2}{ }^{*}$ are predicted to be identical. This process is called "motional averaging regime" (MAR) first introduced by Brooks.[41] In this regime, the transverse relaxivity $R_{2}$ increases with increasing particle size. However for the larger particles, proton diffusion is not the dominant factor for signal decay, as the induced surrounding perturbing field is stronger. Therefore, the relaxation rate $1 / T_{2}$ becomes independent on diffusion, and will not increase with the increasing particle size. Thus, the value for $R_{2}$ is usually smaller than $\mathrm{R}_{2}{ }^{*}$. This process is called "static dephasing regime" (SDR) which was first introduced by Yablonskiy and Haacke.[42] Usually the small sin- gle core magnetic nanoparticles developed by many research groups and commonly used for MRI applications in vivo are in the MAR. This is where larger magnetic nanoparticles possess a larger magnetization and exhibit higher $\mathrm{R}_{2}$ relaxivity.

As described in the $E q .1$, transverse relaxivity $\mathrm{R}_{2}$ is proportional to the magnetic moment. Although magnetism is an intrinsic property of bulk materials, the magnetic properties of nanoparticles are strongly dependent on their size, shape, and surface properties. It has been well demonstrated that the saturation magnetization $M_{s}$ increases with the particle size. A linear relationship is predicted between $\mathrm{M}_{\mathrm{s}}^{1 / 3}$ and $\mathrm{d}^{-1} .[8,13,14,43]$ Therefore, the capability of MRI signal enhancement by nanoparticles correlates directly with the particle size. For example, Cheon and co-workers systematically studied the relationships among size, magnetism, and relaxivity using size uniformed iron oxide nanoparticles. They found that the transverse relaxivity coefficient $\left(R_{2}\right)$ value of the iron oxide nanoparticles gradually increases from 78 $\mathrm{mM}^{-1} \mathrm{~s}^{-1}$ to 106,130 , and to $218 \mathrm{mM}^{-1} \mathrm{~s}^{-1}$ at $1.5 \mathrm{~T}$ as the particle size increases from $4 \mathrm{~nm}$ to 6,9 , and $12 \mathrm{~nm}$ respectively.[44] Many other research groups have also reported on the similar size effect of relaxation rates. $[8,38,45,46]$ However, the $R_{2}$ relaxivity is predicted to approach a plateau as the particle size continues increasing according to the SDR theory.[47] In the case of polyvinylpyrrolidone (PVP) coated IONPs, $\mathrm{R}_{2}$ values increased from $173.37 \mathrm{mM}^{-1} \mathrm{~s}^{-1}$ to 203.86, 239.98 , and $248.89 \mathrm{mM}^{-1} \mathrm{~s}^{-1}$ at $7 \mathrm{~T}$, when the particle size arise from $8 \mathrm{~nm}$ to $23,37,65 \mathrm{~nm}$, respectively. However, it has been reported that the size effect became less significant for the single core IONPs above 50 nm.[31]

Another approach for enhancing magnetic properties for high MRI contrast effect is to fabricate magnetic nanoclusters comprised of small magnetic nanoparticles, as a result, increasing effective magnetic size. Gao and co-workers developed a type of nanoclusters with polymeric micelles encapsulating 4 $\mathrm{nm}$ iron oxide nanoparticles. They demonstrated the dramatic increase of the $R_{2}$ relaxivity $\left(169 \mathrm{Fe} \mathrm{mM}^{-1} \mathrm{~s}^{-1}\right.$ increased from $25.1 \mathrm{Fe} \mathrm{mM}^{-1} \mathrm{~s}^{-1}$ at $1.5 \mathrm{~T}$ ), thus substantially improving the detection sensitivity (at nanomolar concentration) of targeted tissues.[48] Berret et al. investigated the relaxation behaviors of $6.3 \mathrm{~nm}$ IONPs and their nanoclusters. It is found that the $R_{2}$ relaxivity value is noticeably increased with the size of the magnetic clusters. Compared to $39 \mathrm{mM}^{-1} \mathrm{~s}^{-1}$ of bare single IONPs, the transverse relaxivity $R_{2}$ of the IONPs clusters change from $74 \mathrm{mM}^{-1} \mathrm{~s}^{-1}$ to $162 \mathrm{mM}^{-1} \mathrm{~s}^{-1}$ at $0.47 \mathrm{~T}$ as the cluster size increases from $70 \mathrm{~nm}$ to 170 nm.[49] After enwrapped with amphiphilic diblock 
copolymer PEO-b-PyMPS developed by Chen et al, 13-nm IONPs formed clusters with an averaged core size of $50 \mathrm{~nm}$, resulting much higher magnetization saturation and subsequently higher transverse relaxivity $R_{2}$ value as shown in Figure 2.[50] The investigation by Weller and co-workers suggested that homogeneously dispersed nanoparticles satisfy the MAR theory while the clusters embedded in lipid micelles is in agreement with the SDR theory with capability of greatly enhancing contrast in $\mathrm{T}_{2}{ }^{*}$-weighted images.[46]

For the particulate $\mathrm{T}_{1}$ contrast agents, the paramagnetic ions incorporated on the surface of nanoparticles are responsible for the shortening longitudinal relaxation time $T_{1}$. As a result, the relaxivity $R_{1}$ is more dependent on the total surface area of the nanoparticles than on the size of the nanoparticles. Re- cently, Hyeon and co-workers developed $\mathrm{MnO}$ nanoparticles with $\mathrm{T}_{1}$ contrast enhancement and tested in MR imaging of brain, liver, and kidney.[51] They found that the relaxivity $R_{1}$ decreases with the increasing particle size. The $R_{1}$ value changes from 0.37 $\mathrm{mM}^{-1} \mathrm{~s}^{-1}$ to $0.18,0.130 .12 \mathrm{mM}^{-1} \mathrm{~s}^{-1}$ at $3.0 \mathrm{~T}$, as the particle size increases from $7 \mathrm{~nm}$ to $15,20,25 \mathrm{~nm}$. To increase surface-to-volume ratio, they etched $\mathrm{MnO}$ nanoparticles into hollow structures, where more active $\mathrm{Mn}^{2+}$ are exposed to water.[52] As a result, the hollow $\mathrm{MnO}$ nanoparticles exhibited much higher $\mathrm{R}_{1}$ value (1.42 $\left.\mathrm{mM}^{-1} \mathrm{~s}^{-1}\right)$ compared to the solid ones $(0.21$ $\left.\mathrm{mM}^{-1} \mathrm{~s}^{-1}\right)$. With the hollow core-shell configuration, such nanoconstruct could be applied as a drug delivery system with effective MRI contrast enhancement for MRI monitoring drug delivery.
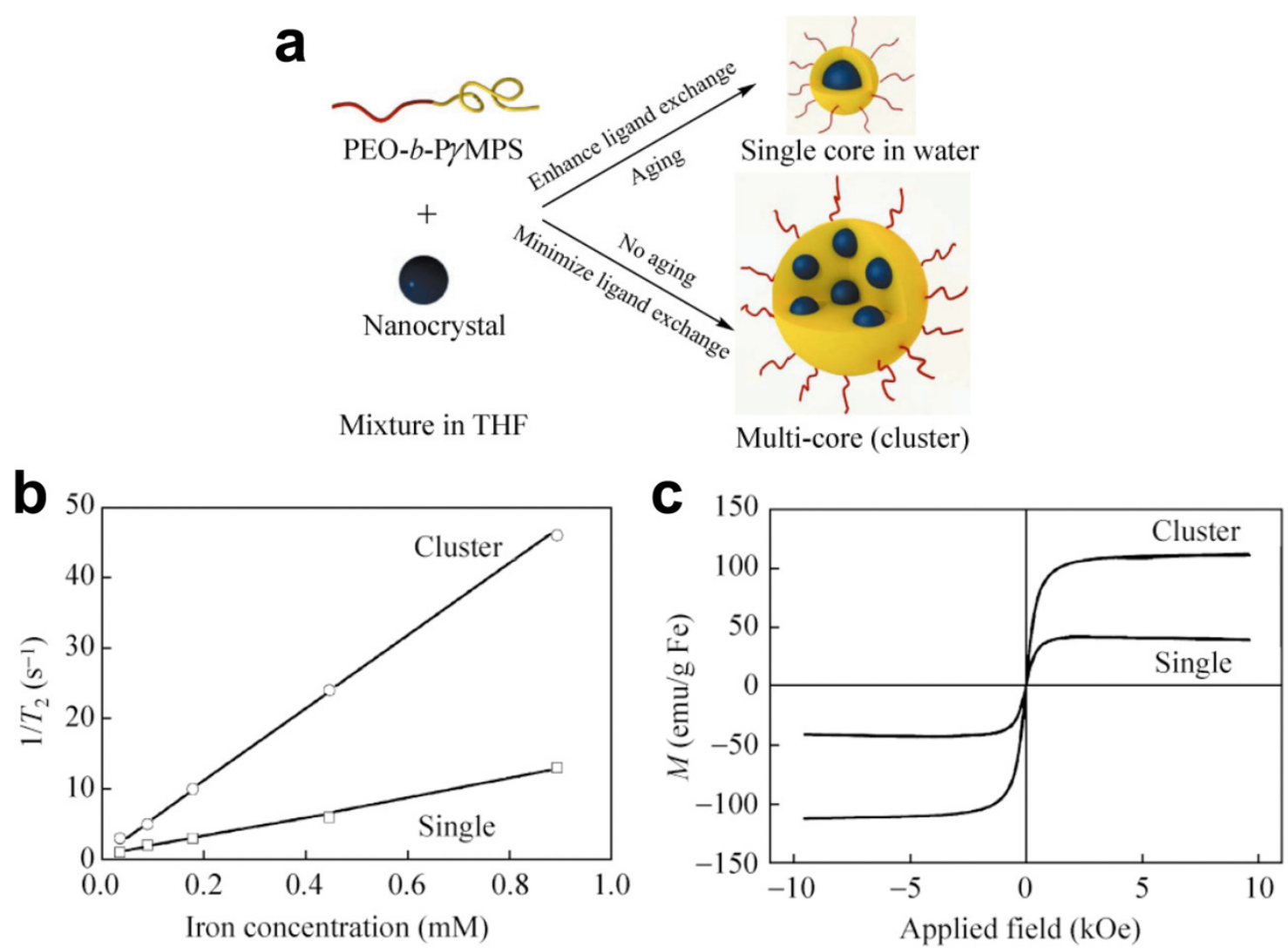

Figure 2 . (a) Schematic illustration of the stabilization of nanoparticles with a single core or a multi-core in water using the amphiphilic diblock copolymer PEO-b-PYMPS. (b) Plots of the linear correlations of transverse relaxation rates $\left(\mathrm{I} / \mathrm{T}_{2}, \mathrm{~s}^{-1}\right)$ of PEO-b-PYMPS-coated monodisperse single core $(\square)$ and clustered $(\circ)$ IONPs against the iron concentration (mmol/L), allowing the transverse relaxivities at 3 Tesla of each nanoparticle formation to be derived from the slopes of the plots. (c) Magnetization of monodisperse single core and clustered IONPs as a function of the applied magnetic field. Adapted with permission from ref [50]. 


\subsection{Nanoparticle Surface Properties Effect on MRI Contrast}

MRI contrast comes from the signal difference between water molecules residing in different environments that are under the effect of magnetic nanoparticles. Because the interactions between water and the magnetic nanoparticles occur primarily on the surface of the nanoparticles, surface properties of magnetic nanoparticles play important roles in their magnetic properties and the efficiency of MRI contrast enhancement. As most biocompatible magnetic nanoparticles developed for in vivo applications need to be stabilized and functionalized with coating materials, the coating moieties can affect the relaxation of water molecules in various forms, such as diffusion, hydration and hydrogen binding. The early investigation carried at by Duan et al suggested that hydrophilic surface coating contributes greatly to the resulted MRI contrast effect.[45] Their study exam- ined the proton relaxivities of iron oxide nanocrystals coated by copolymers with different levels of hydrophilicity including: poly(maleic acid) and octadecene (PMO), poly(ethylene glycol) grated polyethylenimine (PEG-g-PEI), and hyperbranched polyethylenimine (PEI). It was found that proton relaxivities of those IONPs depend on the surface hydrophilicity and coating thickness in addition to the coordination chemistry of inner capping ligands and the particle size. Directly exchange between the multivalent ligands (e.g. PEI and PEG-g-PEI) with the original hydrophobic surface ligand (e.g. oleic acid) facilitates the accessibility of water to the magnetic core. This leads to the rapid exchange and diffusion of water molecules between the bulk phase and the adjacent layer surrounding the particle surface. $10 \mathrm{~nm}$ IONPs coated with high hydrophilic PEI polymer showed the highest $\mathrm{R}_{2}$ and the largest signal drop in $\mathrm{T}_{2}$ weighted fast spin-echo imaging as shown in Figure 3.

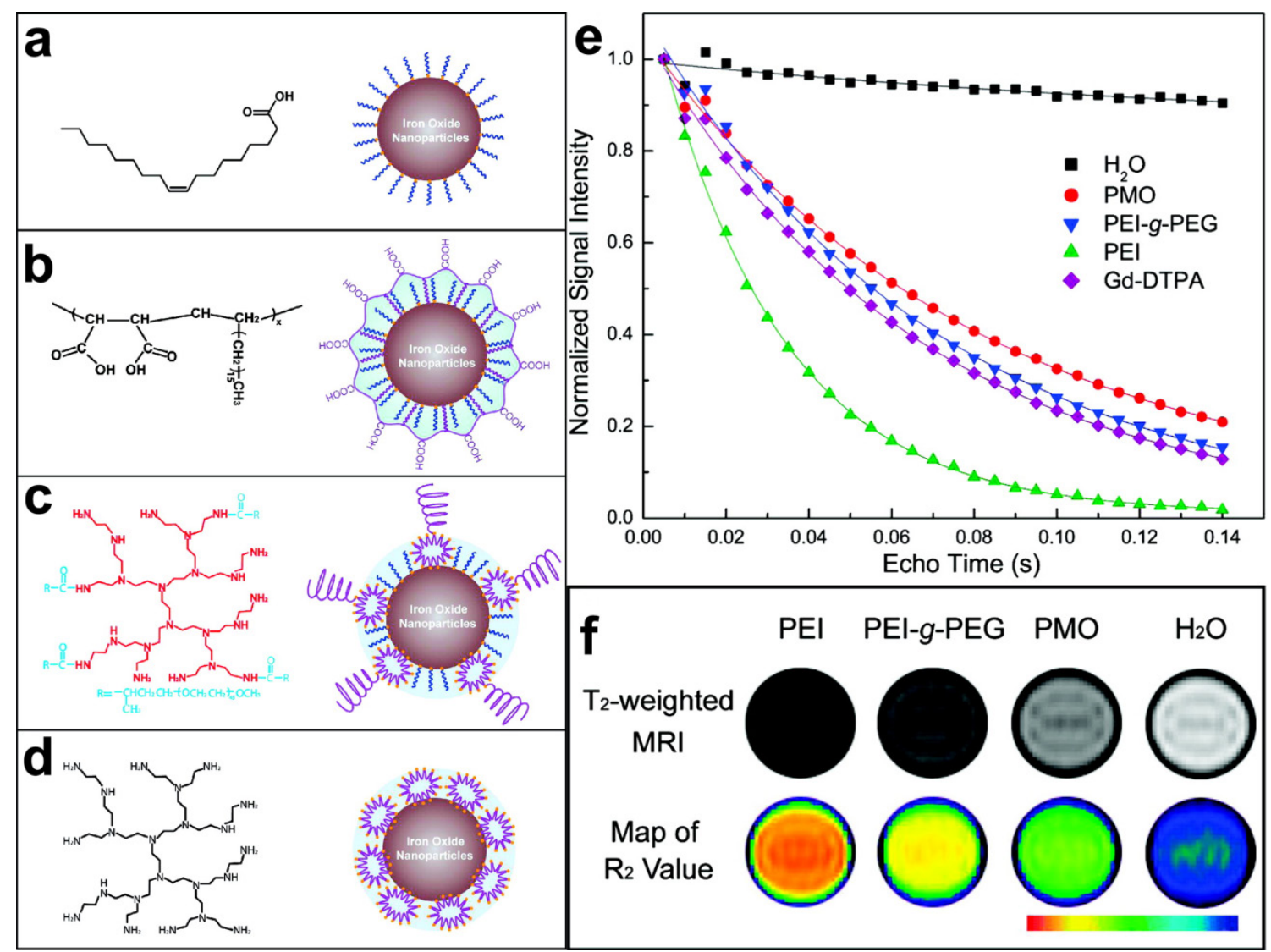

Figure 3. (a-d) Organic polymers for solubilizing hydrophobic iron oxide nanocrystals and schematic structures of polymer-coated iron oxide nanocrystals: (a) oleic acid; (b) copolymer of poly(maleic acid) and octadecene (PMO); (c) poly(ethylene glycol) grated polyethylenimine (PEG-g-PEI), (d) hyperbranched polyethylenimine (PEI). (e) Comparison of traverse relaxation signal intensities $\left(T_{2}\right)$ for pure water, Gd-DTPA (a gadolinium complex), and polymer-coated iron oxide nanoparticles as a function of echo delay time. (f) $T_{2}$-weighted contrasts and color coded $R_{2}$ maps for iron oxide nanocrystals (10 nm core size) with different polymer coatings. Adapted with permission from ref [45]. 
This was almost three times higher than that of PMO coated nanoparticles, which have the lowest hydrophilicity. Meanwhile, the capping ligands that strongly bind to surface iron (e.g. oleic acid) largely influence intrinsic surface spin disorders. This partially offset the size effect arisen from the larger surface area to volume ratios. The proton relaxivity of 30 nm IONPs coated with oleic acid and PMO is 2.6 times that of $10 \mathrm{~nm}$ particles, while the increase is less than $40 \%$ for that capped with PEI.

The thickness of surface coating materials also contributed to the relaxivity and contrast effect of the magnetic nanoparticles. Tong et al systematically modified the core size and polyethylene glycol (PEG) coating of SPIOs for the maximal $\mathrm{R}_{2}$ relaxivity per-particle.[53] Using 1, 2-distearoyl-sn-glycero-3phosphoethanolamine-N-[methoxy (polyethylene glycol)] copolymer (DSPE-mPEG), they were able to control the coating thickness by varying the size of PEG from $500 \mathrm{Da}$ up to $5000 \mathrm{Da}$. Generally, the measured $T_{2}$ relaxation time increases as molecular weight of PEG increases, although the effect of the PEG thickness also interplays with the particle core size. At the optimal condition using $1000 \mathrm{Da}$ of PEG coating $14 \mathrm{~nm}$ core iron oxide nanoparticles, the $R_{2}$ relaxivity of PEG coated SPIO reached a value of 385 $\mathrm{s}^{-1} \mathrm{mM}^{-1}$ at $7 \mathrm{~T}$, more than 2.5 folds higher than that coated with PEG $5000 \mathrm{Da}$. The significant thinner coating thickness of PEG 1000 (hydrodynamic size of $28.4 \mathrm{~nm}$ for $14 \mathrm{~nm}$ core) is considered to be the major factor of the improved relaxivity.

Weller and co-workers investigated the relaxivities of $\mathrm{MnFe}_{2} \mathrm{O}_{4}$ nanoparticles modified through ligand exchange through additional polymeric coating and lipid micellar embedding.[46] The transverse relaxivity, $\mathrm{R}_{2}{ }^{*}$ is greatly higher for the micellar system compared to the polymer-coated particles transferred from same-sized nanoparticles. One explanation for the observed high $R_{2}$ is that the core cluster inside lipid micelles has a larger overall size than the single dispersed polymer-coated nanoparticles. In addition to the discussed composition concern, the surface properties are also important for the responsibility of contrast enhancement. Lipid molecules influence the diffusion of water inside the micelles and in their surroundings. Furthermore, the dephasing proton spins of the lipid molecules also contribute to the signal reduction due to the high viscosity of these molecules.

\section{Novel MRI Methods for Improving the Imaging Contrast, Sensitivity, Detectability and Quantification of Magnetic Nanoparti- cles}

Utilization of contrast agents in MRI leads to hypointense/hyperintense signals in MR images, generating so-called "negative contrast"/"positive contrast". Because magnetic nanoparticles provide various contrast enhancement options, MRI methods and new imaging sequences can be developed and applied based on different contrast mechanisms to improve the detection of engineered magnetic nanoparticle probes. More importantly probes and drug delivery vehicles can be quantified in vivo using MRI. With a better understanding of MRI contrast mechanisms and properties of contrast materials, the design and preparation of engineered magnetic nanoparticles therefore can be specifically "tailored" to take advantage of the MRI methods for the intended imaging applications.

\section{I. Conventional MRI Sequences and Methods for Imaging Magnetic Nanoparticles}

Unlike the conventional small molecule gadolinium chelate contrast agents, magnetic nanoparticle based on MRI probe has strong field strength dependency due to the superparamagnetic nature of magnetic nanoparticles. In a detailed report, Rohrer et al studied and compared various commercially available contrast agents for MRI in terms of their $R_{1}$ and $R_{2}$ relaxivities at magnetic field strengths ranging from $0.47 \mathrm{~T}$ to 4.7T.[54] Their results showed that compared to the Gd-based chelates, contrast agents based on superparamagnetic iron oxide, e.g. Feridex and Resovist, exhibited significant dependencies of relaxivities on the field strengths. Therefore, in preclinical animal imaging research, magnetic nanoparticles offer the best sensitivity as high field MRI instrumentation is typically used. Experiments are often done at the field strengths ranging from $4.7 \mathrm{~T}$ to $11.7 \mathrm{~T}$ for MRI and MR microscopy imaging with different animal models.[55-57]

Most engineered magnetic nanoparticles are known as excellent $T_{2}$ and $T_{2}{ }^{*}$ contrast agents. Magnetic nanoparticles, in a magnetic field, create extremely large microscopic field gradients to diphase nearby protons, and exhibit superb ability in shortening transverse relaxation times $\mathrm{T}_{2}$ and $\mathrm{T}_{2}{ }^{*} \cdot[10,58]$ Therefore, the most commonly used imaging sequences for detecting magnetic nanoparticles are $T_{2}$ and $\mathrm{T}_{2}{ }^{*}$ weighted sequences.[23, 59-62] Spin-echo based $\mathrm{T}_{2}$ weighted imaging provides great anatomic details, and it is insensitive to the susceptibility artifacts because of the property of spin echo readout. On gradient-recalled echo (GRE) images, where $\mathrm{T}_{2}{ }^{*}$ effects dominate, an even stronger decrease in signal intensity of target organs is induced. GRE imaging generates more hypointense contrast in $\mathrm{T}_{2}{ }^{*}$ weighted 
images compared to $T_{2}$ weighted SE images.[60] However, gradient-echo based $\mathrm{T}_{2}{ }^{*}$ weighted imaging is more vulnerable to the susceptibility artifacts rising from the tissue-air interface and iron depositions or other local inhomogeneities in some organs and diseased areas, e.g., bone marrow and lung.

Imaging sequences used for detecting magnetic nanoparticles are mainly categorized as $\mathrm{T}_{2}$ weighted imaging, $\mathrm{T}_{2}$ relaxometry, and $\mathrm{T}_{2}{ }^{*}$ weighted imaging. Although both $\mathrm{T}_{2}$ and $\mathrm{T}_{2}{ }^{*}$ weighted imaging approach offer high sensitivity for detecting magnetic nanoparticles, the main drawback of the negative contrast is its poor contrast when used to study areas that have low background signals. Another drawback is that they are interfered by high magnetic inhomogeneity or susceptibility effect, which also results in the intrinsic tissue signal void. $T_{2}$ and $T_{2}{ }^{*}$ weighted imaging methods are also vulnerable to the "blooming" artifacts and partial volume effects making accurate localization and quantitative imaging difficult.[59, 63] Furthermore, higher magnetic susceptibility and stronger $T_{2}$ and $T_{2}{ }^{*}$ effect from large sized magnetic nanoparticles may not be translated to better negative contrast in $\mathrm{T}_{2}$ and $\mathrm{T}_{2}{ }^{*}$ weighted imaging. The contrast may not improve further as the level of signal decrease cannot get beyond voiding the signal from its original level.[64] To overcome these limitations of negative contrast of conventional MRI methods, significant efforts have been made in developing alternative imaging methods that are capable of enhancing the signal or generating bright and positive contrast to improve the detection of magnetic nanoparticles.

\subsection{Turning the Contrast Opposite - "Bright Imaging" of Magnetic Nanoparticles}

One attractive approach for generating positive contrast is the off-resonance excitation methods. Cunningham et al.[65] and Balchandani et al.[66] proposed to visualize magnetic nanoparticle affected protons by selectively exciting the off-resonance spins surrounding the labeled cells to generate positive contrast. In addition, inversion-recovery with on-resonant water suppression (IRON) may be used in conjunction with the spectrally selective RF pulses.[67] Alternatively, fast low-angle positive contrast steady-state free precession imaging (FLAPS) is able to generate positive contrast from the off-resonant spins while suppressing the on-resonant spins in a flip-angle-dependent manner. Because the background suppression is controllable, co-registration of the bright signal as a result of the contrast enhancement by the magnetic nanoparticles and the anatomic details can be made in the same image.[68, 69]
Alternatively, Zurkiya et al. reported an off-resonance saturation (ORS) method to produce positive contrast.[70] In this method, an RF pulse is designed to be applied at an off-resonance frequency and bulk water protons are imaged with and without the presence of the ORS pulse. This method relies on diffusion saturation transfer to reduce the on-resonance MRI signal. This is similar to chemical exchange saturation transfer. The positive contrast was obtained by taking the ratio or subtraction of the images with and without the saturation. Using this ORS approach, Khemtong et al successfully turned "ON" the tumor-targeted superparamagnetic polymeric micelles (SPPM) in vivo, in contrast to the "negative" contrast from the $\mathrm{T}_{2}{ }^{*}$ weighted GRE imaging (Figure 4).[18]

Recently Zhou et al. developed a different approach to exploit the positive contrast with a relatively new technique called sweep imaging with Fourier transformation (SWIFT). [71] SWIFT is based on a frequency-swept excitation with virtually simultaneous signal acquisition with the radiofrequency excitation. Therefore it is particularly well suited to image objects with extremely fast transverse relaxation rates such as IONPs. Unlike the off-resonance excitation methods, which generate images that contain signal only in areas that correspond to the locations of IONPs and therefore need an extra image acquisition to provide background anatomic details, SWIFT approach generates IONP-related signal as positive contrast in the imaginary component of the image. The magnitude image contains all the anatomic details of the heart (Figure 5).

Owing to the predominant $T_{2}$ or $T_{2}{ }^{*}$ effects over the usual dipole-dipole relaxation mechanism that affects both $T_{1}$ and $T_{2}$, IONPs are usually treated as $T_{2}$ or $\mathrm{T}_{2}{ }^{*}$ contrast agents. On the other hand, IONPs also exhibit a high longitudinal $\mathrm{R}_{1}$ relaxivity providing intrinsic signal enhancement on $T_{1}$ weighted images.[72, 73] However, conventional imaging sequences cannot fully take advantage of such strong $\mathrm{T}_{1}$ weighted contrast effect because of the predominant $\mathrm{T}_{2}$ and spin dephase effects of IONPs. Ultrashort echo time (UTE) imaging is capable of imaging materials with extremely short $\mathrm{T}_{2}$ and very fast signal decay.[74] With very short echo time, typically below $0.1 \mathrm{~ms}$, UTE imaging allows for signal acquisition with little $\mathrm{T}_{2}$ influence. In a recent study by Zhang et al, UTE imaging was applied to generate $T_{1}$ weighted positive contrast enhancement for IONPs with different core sizes and concentrations in phantoms (Figure 6).[19]

The UTE imaging was also demonstrated to yield positive contrast for U87MG human glioblastoma cells targeted with an IONPs conjugated with a 
small peptide ligand of arginine-glycine-aspartic acid (RGD). This has a high affinity to the cells overexpressing $\alpha_{v} \beta_{3}$ integrin (Figure 7).[19]

\subsection{MRI Quantification of Magnetic Nanoparti- cles}

Ideally, the amount of IONPs should be quantified so that longitudinal studies can be performed for monitoring the delivery of drug delivery, tracking the nanoparticle distributions, and evaluating as well as comparing the treatment responses. Quantitative relaxometry or mapping of relaxivity values over the imaged area is a common and straightforward approach. The relaxometry methods usually use the magnitude information from multi-echo MR images and quantify the concentration of IONPs by fitting the relaxation rate of the IONPs within the tissue. By acquiring data with a multi-echo SE sequence and fitting the signal intensities to the exponential $\mathrm{T}_{2}$ decay equation, Hogemann et al. calculated $\mathrm{T}_{2}$ maps of a limited library of peptide-nanoparticles conjugates for cell uptake on 1920 samples of six 384 well plates (each contains $16 \times 20$ wells). This demonstrated that $\mathrm{T}_{2}$ mapping provided exceedingly accurate measurements and receptor binding/internalization data as validated by radioactive assays.[75]
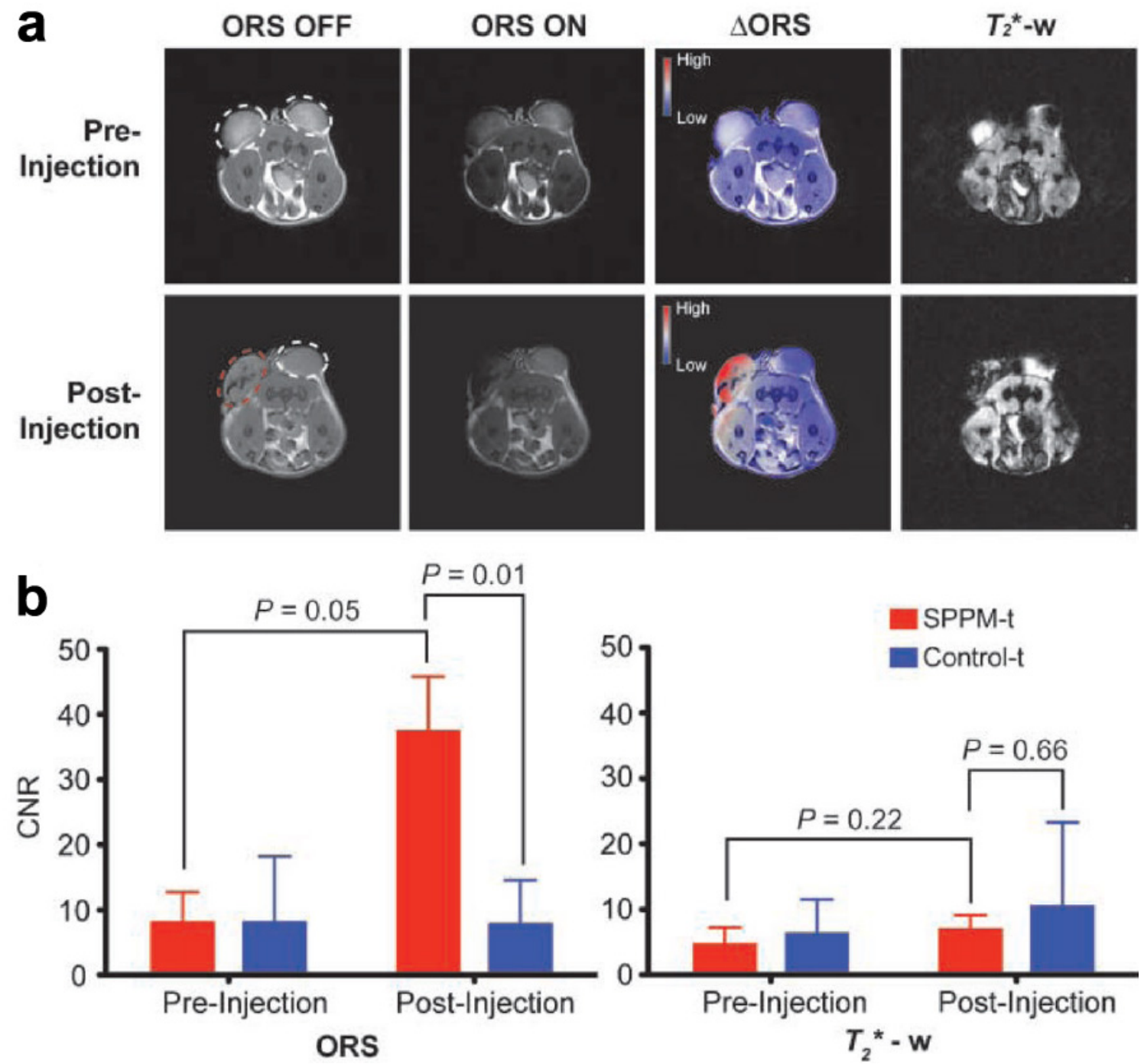

Figure 4. Comparison of ORS and $\mathrm{T}_{2}{ }^{*}$ weighted imaging of SPPM nanoprobes $(20 \mu \mathrm{L}, 0.25 \mathrm{mg} / \mathrm{mL})$ intratumorally injected inside an A549 tumor xenograft in a mouse. (a) ORS and $\mathrm{T}_{2}{ }^{*}$ weighted images of the transverse section of a representative mouse bearing two tumor xenografts (one for SPPM injection (red dashed circle), the other as SPPM-free control) before and after the injection of SPPM. The $\triangle O R S$ images were obtained by pixel-by-pixel subtraction of ORS ON images from ORS OFF images and overlaid with ORS OFF images. (b) Statistical comparison of CNRs of SPPM-injected versus SPPM-free tumors by the ORS and $\mathrm{T}_{2}{ }^{*}$ weighted methods. Reprinted with permission from ref [18]. 

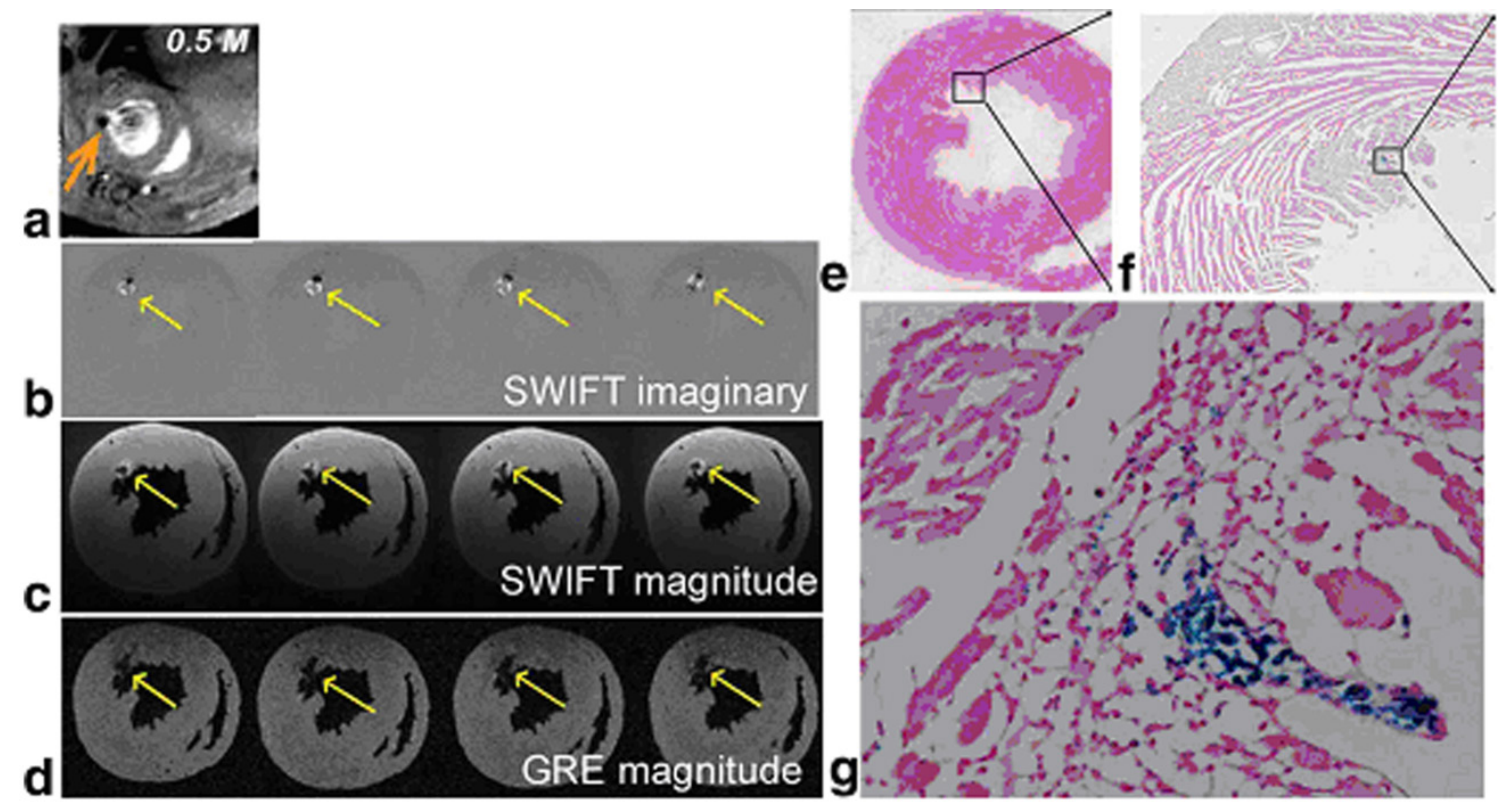

Figure 5. (a) In vivo GRE image; (b) ex vivo SWIFT imaginary and (c) magnitude; (d) ex vivo GRE magnitude images obtained from a heart receiving 0.5 M IONP-labeled stem cells. Arrows indicate location of cells. (e-g) Prussian blue staining of the heart sections at the corresponding level of MR images. Magnified views of the box in (e) and (f) are presented in (f) and $(g)$, respectively. Reprinted with permission from ref [7I].

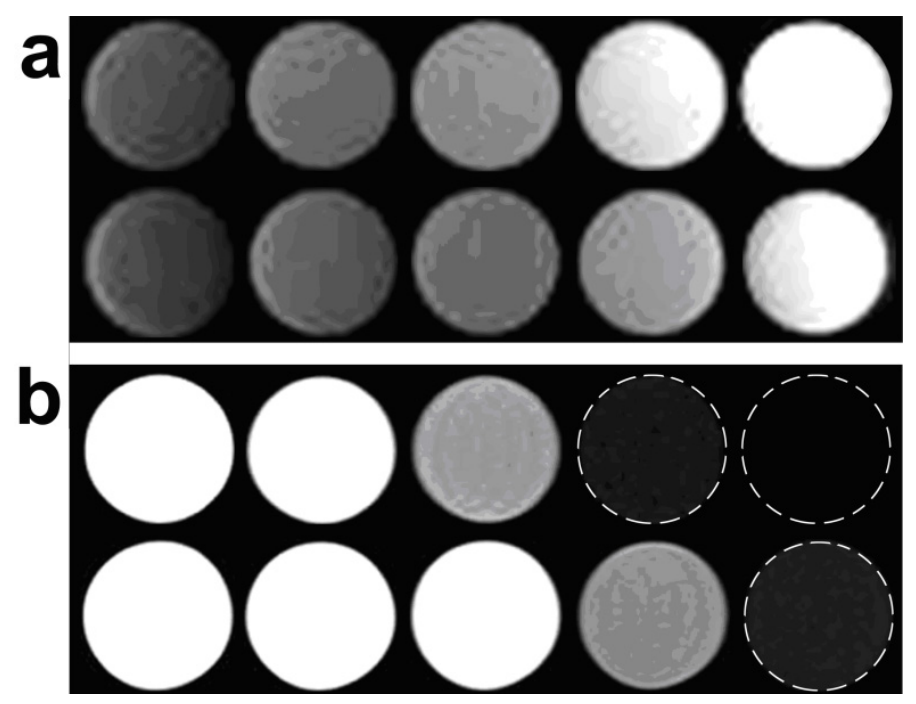

Figure 6. Comparison of $T_{1}$-weighted UTE and conventional $T_{2}$-weighted TSE images of IONPs; (a) $T_{1}$ weighted UTE images of selected IONP samples. (b) Conventional $T_{2}$ weighted TSE images of the same samples. In each image panel, the top row is the IONP samples with a core size of $25.4 \mathrm{~nm}$ and the bottom row is the IONP samples with a core size of 8.7 $\mathrm{nm}$, and the concentrations are corresponding to level I (left most) to level 5 (right most). UTE images have lower in plane resolution $\left(1.1 \times 1.1 \mathrm{~mm}^{2}\right.$ isotropic) than that of $\mathrm{T}_{2}$ weighted TSE images $\left(0.7 \times 0.7 \mathrm{~mm}^{2}\right.$ isotropic). Reprinted with permission from ref [19]. 


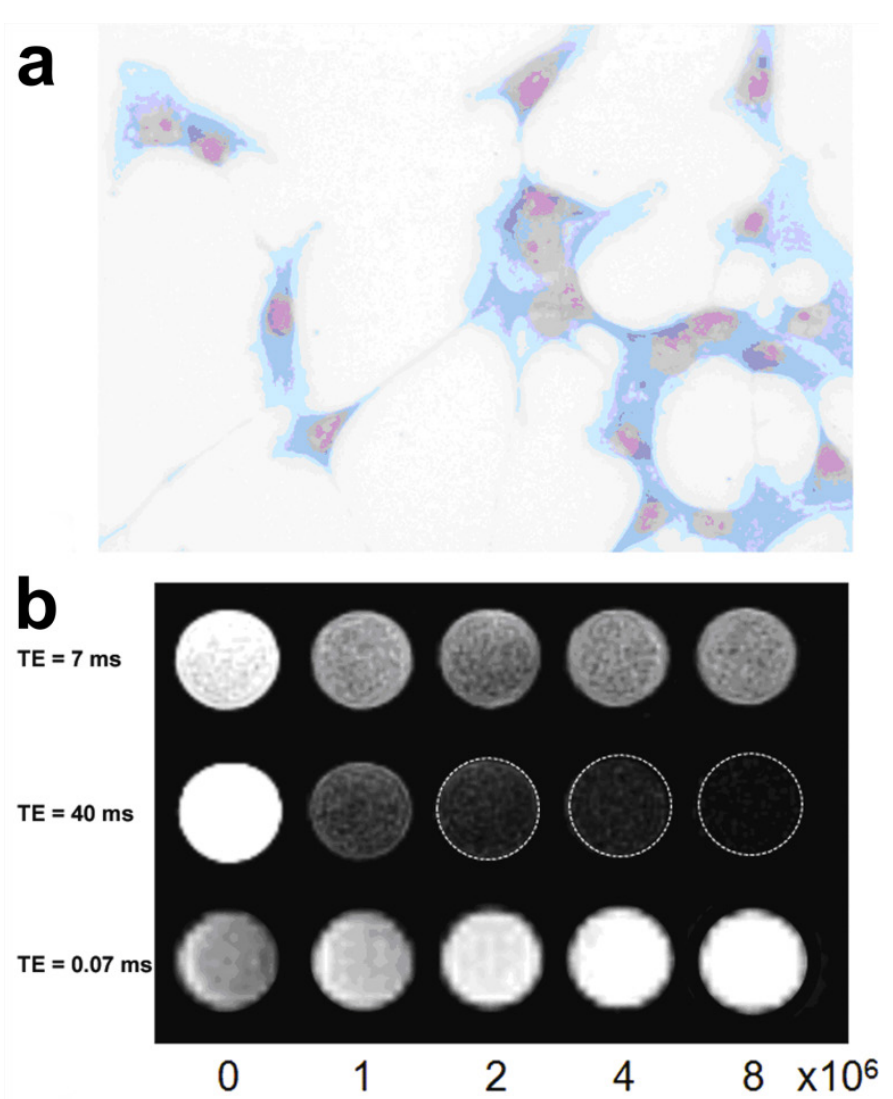

Figure 7. (a) Prussian blue staining confirmed the presence of tumor integrin targeted RGD-IONP conjugates in U87MG human glioblastoma cells. (b) Comparison of moderately $T_{1}$ weighted TSE images (top), moderately $\mathrm{T}_{2}$ weighted TSE images (middle) and $T_{1}$ weighted UTE images (bottom) of cell phantoms containing $0,1,2,4$ and $8 \times 10^{6}$ U87MG glioblastoma cells bound with RGD-IONP conjugates. Cells were embedded in $2 \%$ agarose gel. Reprinted with permission from ref [19].

Lee et al visualized small tumors implanted in a mouse with their ultra-sensitive magnetism-engineered iron oxide (MEIO), and he used $\mathrm{T}_{2} / \mathrm{R}_{2}$ mapping to evaluate the enhancement and sensitivity of their approach.[8] In a recent study, Liu et al used T2 mapping to show the over expression of ferritin for the demonstration of metalloprotein reporter gene-based noninvasive monitoring of embryonic stem cells in vivo.[76] Because $\mathrm{T}_{2}$ relaxometry methods applied a "shot-gun" approach to capture $\mathrm{T}_{2}$ decay trend with multiple echo times in one scan. It has better sensitivity than conventional $\mathrm{T}_{2}$ weighted imaging which uses only one echo time that may not be an optimal one for maximal contrast. Since the $T_{2}$ relaxivity is closely correlated with the concentration of the magnetic nanoparticles given specific physic-chemical properties, the quantification of magnetic nanoparticles can be accomplished.

Alternatively, $\mathrm{T}_{2}{ }^{*}$ mapping can be obtained using multi-echo GRE sequence. Because $\mathrm{T}_{2}{ }^{*}$ has a greater sensitivity compared $\mathrm{T}_{2}, \mathrm{~T}_{2}{ }^{*}$ mapping is expected to be more sensitive than $T_{2}$ mapping. How- ever, for IONPs with extremely short $\mathrm{T}_{2}{ }^{*}$ relaxation times (e.g. $\leq 3 \mathrm{~ms}$ ), the signal decay may be too rapid for the regular GRE sequence. In a recent study, Liu et al. developed a shifted spin echo (SSE) sequence to allow the acquisition of ultrashort $\mathrm{T}_{2}{ }^{*}$ relaxometry.[77] Combined with the regular multi-echo GRE sequence, their approach was able to provide quantification of highly concentrated iron-labeled cells from direct cell transplantation with had extremely short $\mathrm{T}_{2}{ }^{*}$ (Figure 8).

Model-based methods provide alternative ways to quantify magnetic nanoparticles. With prior knowledge of empirical physics and mathematics models and the MRI data acquired corresponding to the model design, the model-based methods quantify the concentration of IONPs by fitting the experimental MR data in the models. For example, Zurkiya et al proposed a simple model for the ORS method in their original publication which shed the light of quantifying magnetic nanoparticles using the ORS method.[70] In addition, Khemtong et al further reported a theoretical model of ORS contrast and its 
experimental validation by SPPM with precisely controlled structural properties. A simplified mathematical expression was proposed that allowed for quantitative approximation of ORS contrast for a given SPPM solution with known $\mathrm{T}_{1}$ and $\mathrm{T}_{2}$ values.[78]

Very recently, a phase gradient mapping (PGM) method, namely finite perturber method, was proposed by Langley et al.[79] and Zhao et al.[80] This method models magnetic field inhomogeneities by approximating regions containing IONPs as ensembles of magnetic dipoles, and it fits the magnetic field of the model to the experimental magnetic field inhomogeneities identified in the acquired phase maps. This method was verified using data acquired from in vivo mouse models, and it was able to give estimations of the concentration within
IONP-labeled tumors that were reasonably close to the known concentration.[79]

Another promising approach to quantify magnetic nanoparticles arises from the UTE method. In the study performed by Zhang et al, increases of the signal intensity and positive contrast of IONPs in UTE images were found to be correlated to the increases of the size and iron concentration of IONPs (Figure 9).[19] Specifically, for a certain core size of the magnetic nanoparticle, excellent linear correlation was found between the signal intensity of UTE images and the concentration of the magnetic nanoparticle (Figure $9 b$ ). This linear relationship can possibly be used as a simple and straightforward model to quantify magnetic nanoparticles according to the intensity of UTE images after calibration.
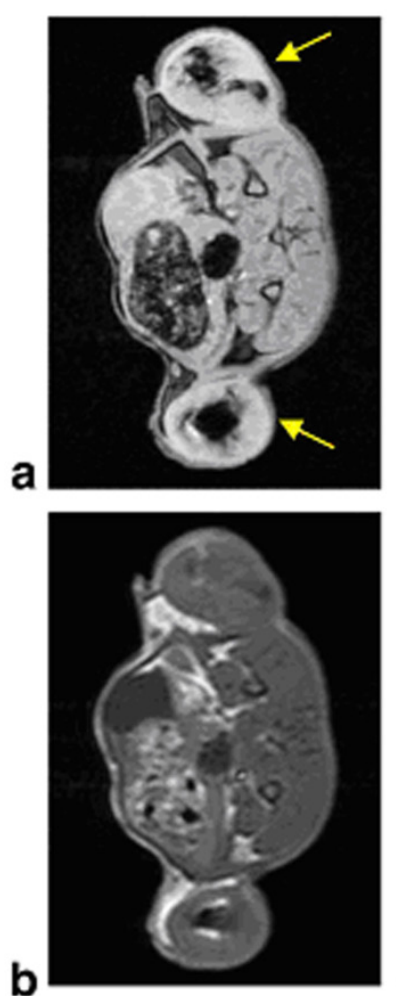
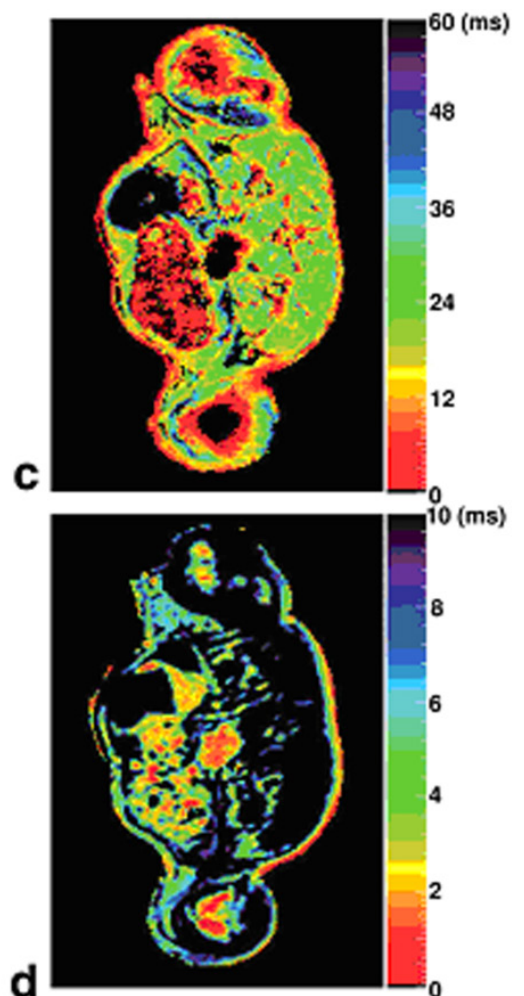
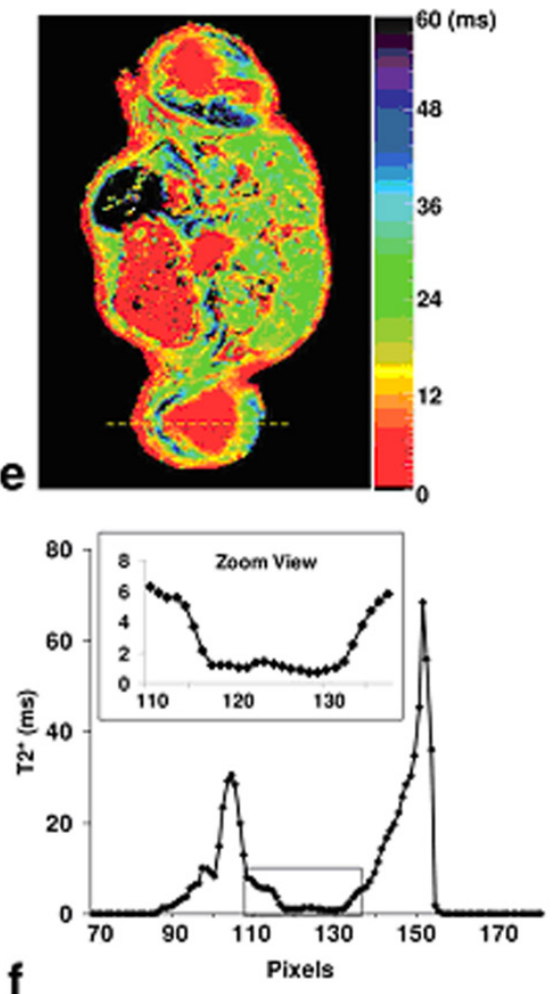

Figure 8. (a) GRE image of an axial slice of a rat with IONP-labeled flank tumors (yellow arrows); (b) SE image of the same slice; (c) Regular $\mathrm{T}_{2}{ }^{*}$ map from the multi-echo GRE sequence; (d) SSE ultrashort $\mathrm{T}_{2}{ }^{*}$ map; (e) SSE ultrashort $\mathrm{T}_{2}{ }^{*}$ map overlaid on the regular $\mathrm{T}_{2}{ }^{*}$ map; (f) Profile of the yellow line in (e). The dark regions within the tumors in (a) and (c) corresponded to highly concentrated IONP-labeled cells. The multi-echo $\mathrm{GRE} \mathrm{T}_{2}{ }^{*}$ mapping technique failed to detect any signal from the center of the tumors (dark circle) but the SSE ultrashort $\mathrm{T}_{2}{ }^{*}$ mapping technique adequately characterized the $\mathrm{T}_{2} *$ relaxation times. The zoomed view in (f) clearly illustrated that $\mathrm{T}_{2}{ }^{*}$ values in the tumor center were in the range of $0 \sim 2 \mathrm{~ms}$. Reprinted with permission from ref [77]. 

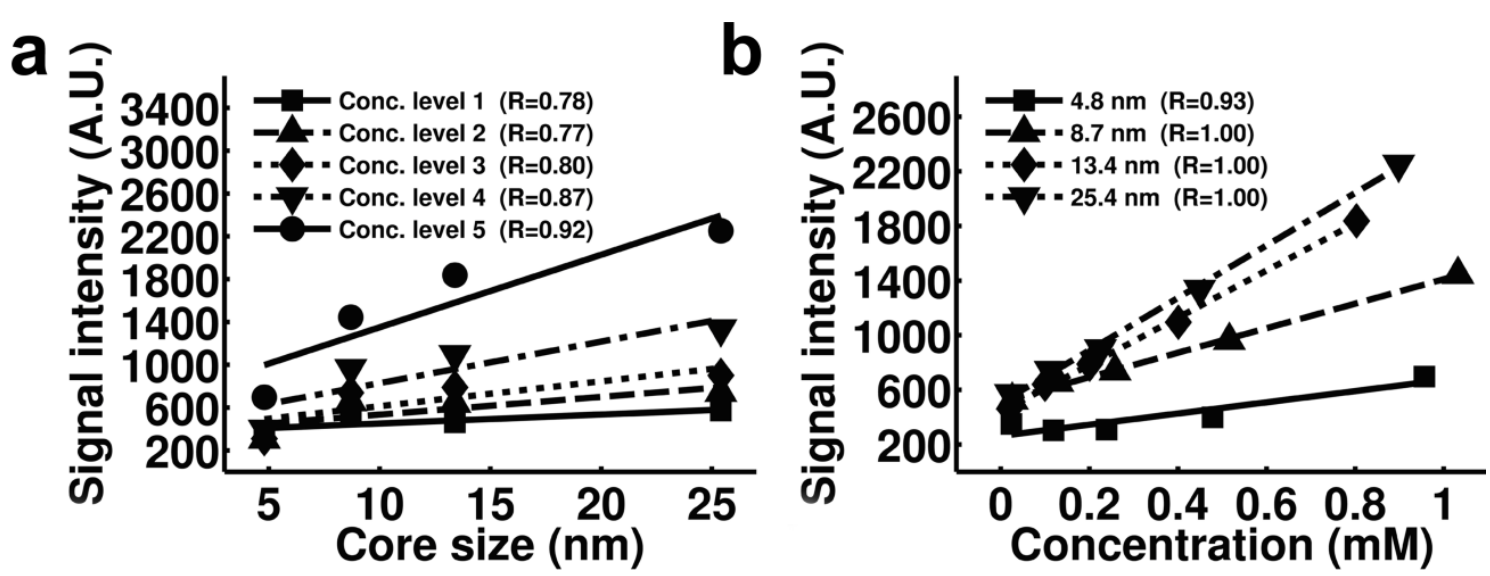

Figure 9. Plots of the signal intensity of $T_{1}$ weighted UTE images of IONP samples with different core sizes (a) and concentrations (b) show stronger positive contrast with higher iron concentrations and larger core sizes. Pearson correlation coefficients of each plot are indicated in the figure legends. Signal intensity is in arbitrary unit (marked as A.U. in the figures). Reprinted with permission from ref [19].

\section{Conclusion}

Much progress has taken place in the theranostic applications of engineered magnetic nanoparticles, especially in MR imaging technologies and nanomaterials development. As the feasibilities of magnetic nanoparticles for molecular imaging and drug delivery have been demonstrated by a great number of studies in the past decade, MRI guiding and monitoring techniques are desired to improve the disease specific diagnosis and efficacy of therapeutics. Continuous effort and development are expected to be focused on further improvement of the sensitivity and quantifications of magnetic nanoparticles in vivo for theranostics in future.

The new design and preparation of magnetic nanoparticles need to carefully consider the parameters determining the relaxivities of the nanoconstructs. Sensitive and reliable MRI methods have to be established for the quantitative detection of magnetic nanoparticles. The new generations of magnetic nanoparticles will be made not only based on the new chemistry and biological applications, but also with combined knowledge of contrast mechanisms and MRI technologies and capabilities. As new magnetic nanoparticles are available for theranostic applications, it is anticipated that new contrast mechanism and MR imaging strategies can be developed based on the novel properties of engineered magnetic nanoparticles.

\section{Abbreviations}

IONPs: iron oxide nanoparticles; MRI: magnetic resonance imaging; SE: spin echo; TSE: turbo spin echo; GRE: gradient echo; ORS: off-resonance saturation; UTE: ultrashort echo time; SPIO: superparamagnetic iron oxide nanoparticles; USPIO: ultrasmall superparamagnetic iron oxide nanoparticles; uPA: urokinase type plasminogen activator; EGFR: anti-epidermal growth factor receptor; ATF: amino-terminal fragment of urokinase type plasminogen activator; uPAR: uPA receptor; NIRF: near-infrared fluorescence; PET: positron emission tomography; HAS: human serum albumin; EPR: enhanced permeability and retention; Nos: noscapine; hATF: human ATF; siRNA: small interfering RNA; MAR: motional averaging regime; SDR: static dephasing regime; PVP: polyvinylpyrrolidone; PMO: poly(maleic acid) and octadecene; PEG-g-PEI: poly(ethylene glycol) grated polyethylenimine; PEI: polyethylenimine; PEG: polyethylene glycol; DSPE-mPEG: 1, 2-distearoyl-sn-glycero-3-phosphoethanolamine-N-[ methoxy (polyethylene glycol)] copolymer; IRON: inversion-recovery with on-resonant water suppression; FLAPS: fast low-angle positive contrast steady-state free precession imaging; SPPM: superparamagnetic polymeric micelles; SWIFT: sweep imaging with Fourier transformation; RGD: arginine-glycine-aspartic acid; MEIO: magnetism-engineered iron oxide; SSE: shifted spin echo; PGM: phase gradient mapping.

\section{Acknowledgements}

This work was supported in part by the following research grants from the National Institute of Health: P50CA128301-03 (HM and LY), U01CA151810-01 (LY and HM) and 1R01CA154846-01A1 (HM and LY). 


\section{Conflict of Interest}

The authors have declared that no conflict of interest exists.

\section{References}

1. Gupta AK, Gupta M. Synthesis and surface engineering of iron oxide nanoparticles for biomedical applications. Biomaterials. 2005; 26: 3995-4021.

2. Laurent S, Forge D, Port M, Roch A, Robic C, Elst LV, Muller RN. Magnetic iron oxide nanoparticles: Synthesis, stabilization, vectorization, physicochemical characterizations, and biological applications. Chem Rev. 2008; 108: 2064-110.

3. Gao J, Gu H, Xu B. Multifunctional Magnetic Nanoparticles: Design, Synthesis, and Biomedical Applications. Acc Chem Res. 2009; 42: 1097-107.

4. Xie J, Huang J, Li X, Sun S, Chen X. Iron Oxide Nanoparticle Platform for Biomedical Applications. Curr Med Chem. 2009; 16: $1278-94$

5. Kim BYS, Rutka JT, Chan WCW. Current Concepts: Nanomedicine. New Engl J Med. 2010; 363: 2434-43.

6. Xie J, Lee S, Chen X. Nanoparticle-based theranostic agents. Adv Drug Delivery Rev. 2010; 62: 1064-79.

7. Xie J, Liu G, Eden HS, Ai H, Chen X. Surface-Engineered Magnetic Nanoparticle Platforms for Cancer Imaging and Therapy. Acc Chem Res. 2011; 44: 883-92.

8. Lee JH, Huh YM, Jun Yw, Seo Jw, Jang Jt, Song HT, Kim S, Cho EJ, Yoon HG, Suh JS, Cheon J. Artificially engineered magnetic nanoparticles for ultra-sensitive molecular imaging. Nat Med. 2007; 13: 95-9.

9. Bulte J, Brooks R. Magnetic nanoparticles as contrast agents for MR imaging. In: Bulte J, Brooks R, ed. Scientific and clinical applications of magnetic carriers New York: Plenum Press; 1997: 527-43.

10. Bulte JWM, Brooks RA, Moskowitz BM, Bryant LH, Frank JA. Relaxometry and magnetometry of the MR contrast agent MION-46L. Magn Reson Med. 1999; 42: 379-84.

11. Shubayev V, Pisanic TR, Jin S. Magnetic nanoparticles for theragnostics. Adv Drug Delivery Rev. 2009; 61: 467-77.

12. Laurent S, Bridot JL, Elst LV, Muller RN. Magnetic iron oxide nanoparticles for biomedical applications. Future Med Chem. 2010; 2: 427-49.

13. Jun YW, Lee JH, Cheon J. Chemical design of nanoparticle probes for high-performance magnetic resonance imaging. Angew Chem Int Ed. 2008; 47: 5122-35.

14. Na HB, Song IC, Hyeon T. Inorganic Nanoparticles for MRI Contrast Agents. Adv Mater. 2009; 21: 2133-48.

15. Veiseh O, Gunn JW, Zhang M. Design and fabrication of magnetic nanoparticles for targeted drug delivery and imaging. Adv Drug Delivery Rev. 2010; 62: 284-304.

16. Girard OM, Du J, Agemy L, Sugahara KN, Kotamraju VR, Ruoslahti E, Bydder GM, Mattrey RF. Optimization of Iron Oxide Nanoparticle Detection Using Ultrashort Echo Time Pulse Sequences: Comparison of T1, T2*, and Synergistic T1-T2* Contrast Mechanisms. Magn Reson Med. 2011; 65: 1649-60.

17. Sosnovik DE, Nahrendorf M, Weissleder R. Magnetic nanoparticles for MR imaging: agents, techniques and cardiovascular applications. Basic Res Cardiol. 2008; 103: 122-30.

18. Khemtong C, Kessinger CW, Ren J, Bey EA, Yang SG, Guthi JS, Boothman DA, Sherry AD, Gao J. In vivo Off-Resonance Saturation Magnetic Resonance Imaging of alpha(v)beta(3)-Targeted Superparamagnetic Nanoparticles. Cancer Res. 2009; 69: 1651-8.

19. Zhang L, Zhong X, Wang L, Chen H, Wang YA, Yeh J, Yang L, Mao H. T1-Weighted Ultrashort Echo Time Method for Positive
Contrast Imaging of Magnetic Nanoparticles and Cancer Cells Bound With the Targeted Nanoparticles. J Magn Reson Imaging. 2011; 33: 194-202.

20. Josephson L, Lewis J, Jacobs P, Hahn PF, Stark DD. The Effects of Iron-oxides on Porton Relaxivity. Magn Reson Imaging. 1988; 6: 647-53.

21. Stark DD, Weissleder R, Elizondo G, Hahn PF, Saini S, Todd LE, Wittenberg J, Ferrucci JT. Superparamagnetic Iron Oxide: Clinical Application as a Contrast Agent for MR Imaging of the Liver. Radiology. 1988; 168: 297-301.

22. Bulte JWM, Zhang SC, van Gelderen P, Herynek V, Jordan EK, Duncan ID, Frank JA. Neurotransplantation of magnetically labeled oligodendrocyte progenitors: Magnetic resonance tracking of cell migration and myelination. Proc Natl Acad Sci USA. 1999; 96: 15256-61.

23. Bulte JWM, Douglas T, Witwer B, Zhang SC, Strable E, Lewis BK, Zywicke H, Miller B, van Gelderen P, Moskowitz BM, Duncan ID, Frank JA. Magnetodendrimers allow endosomal magnetic labeling and in vivo tracking of stem cells. Nat Biotechnol. 2001; 19: 1141-7.

24. Frank JA, Miller BR, Arbab AS, Zywicke HA, Jordan EK, Lewis BK, Bryant LH, Bulte JWM. Clinically applicable labeling of mammalian and stem cells by combining; Superparamagnetic iron oxides and transfection agents. Radiology. 2003; 228: 480-7.

25. Bulte JWM, Kraitchman DL. Iron oxide MR contrast agents for molecular and cellular imaging. NMR Biomed. 2004; 17: 484-99.

26. de Vries IJM, Lesterhuis WJ, Barentsz JO, Verdijk P, van Krieken JH, Boerman OC, Oyen WJG, Bonenkamp JJ, Boezeman JB, Adema GJ, Bulte JWM, Scheenen TWJ, Punt CJA, Heerschap A, Figdor CG. Magnetic resonance tracking of dendritic cells in melanoma patients for monitoring of cellular therapy. Nat Biotechnol. 2005; 23: 1407-13.

27. Yang L, Peng X-H, Wang YA, Wang X, Cao Z, Ni C, Karna P, Zhang X, Wood WC, Gao X, Nie S, Mao H. Receptor-Targeted Nanoparticles for In vivo Imaging of Breast Cancer. Clin Cancer Res. 2009; 15: 4722-32.

28. Yang L, Mao H, Wang YA, Cao Z, Peng X, Wang X, Duan $\mathrm{H}, \mathrm{Ni}$ C, Yuan Q, Adams G, Smith MQ, Wood WC, Gao X, Nie S. Single Chain Epidermal Growth Factor Receptor Antibody Conjugated Nanoparticles for in vivo Tumor Targeting and Imaging. Small. 2009; 5: 235-43.

29. Qiao J, Li S, Wei L, Jiang J, Long R, Mao H, Wei L, Wang L, Yang H, Grossniklaus HE, Liu Z-R, Yang JJ. HER2 Targeted Molecular MR Imaging Using a De Novo Designed Protein Contrast Agent. Plos One. 2011;: 6.

30. Yu MK, Park J, Jon S. Targeting Strategies for Multifunctional Nanoparticles in Cancer Imaging and Therapy. Theranostics. 2012; 2: 3-44.

31. Xie J, Chen K, Huang J, Lee S, Wang J, Gao J, Li X, Chen X. PET/NIRF/MRI triple functional iron oxide nanoparticles. Biomaterials. 2010; 31: 3016-22.

32. Huang J, Xie J, Chen K, Bu L, Lee S, Cheng Z, Li X, Chen X. HSA coated $\mathrm{MnO}$ nanoparticles with prominent MRI contrast for tumor imaging. Chem Comm. 2010; 46: 6684-6.

33. Louie AY. Multimodality Imaging Probes: Design and Challenges. Chem Rev. 2010; 110: 3146-95.

34. Abdalla MO, Karna P, Sajja HK, Mao H, Yates C, Turner T, Aneja R. Enhanced noscapine delivery using uPAR-targeted optical-MR imaging trackable nanoparticles for prostate cancer therapy. J Controlled Release. 2011; 149: 314-22.

35. Hadjipanayis CG, Machaidze R, Kaluzova M, Wang L, Schuette AJ, Chen H, Wu X, Mao H. EGFRvIII Antibody-Conjugated Iron Oxide Nanoparticles for Magnetic Resonance Imaging-Guided Convection-Enhanced Delivery and Targeted Therapy of Glioblastoma. Cancer Res. 2010; 70: 6303-12. 
36. Koenig SH, Kellar KE. Theory of 1/T1 and 1/T2 NMRD Profiles of Solutions of Magnetic Nanoparticles. Magn Reson Med. 1995; 34: 227-33.

37. Jang Jt, Nah H, Lee JH, Moon SH, Kim MG, Cheon J. Critical Enhancements of MRI Contrast and Hyperthermic Effects by Dopant-Controlled Magnetic Nanoparticles. Angew Chem Int Ed. 2009; 48: 1234-8.

38. Seo WS, Lee JH, Sun X, Suzuki Y, Mann D, Liu Z, Terashima M, Yang PC, McConnell MV, Nishimura DG, Dai $H$. $\mathrm{FeCo} /$ graphitic-shell nanocrystals as advanced magnetic-resonance-imaging and near-infrared agents. Nat Mater. 2006; 5: 971-6.

39. Hacliipanayis CG, Bonder MJ, Balakrishanan S, Wang X, Mao H, Hadjipanayis GC. Metallic Iron Nanoparticles for MRI Contrast Enhancement and Local Hyperthermia. Small. 2008; 4: 1925-9.

40. Hao R, Xing R, Xu Z, Hou Y, Gao S, Sun S. Synthesis, Functionalization, and Biomedical Applications of Multifunctional Magnetic Nanoparticles. Adv Mater. 2010; 22: 2729-42.

41. Brooks RA, Moiny F, Gillis P. On T2-shortening by weakly magnetized particles: The chemical exchange model. Magn Reson Med. 2001; 45: 1014-20.

42. Yablonskiy DA, Haacke EM. Theory of NMR signal behavior in magnetically inhomogeneous tissues: the static dephasing regime. Magn Reson Med. 1994; 32: 749-63.

43. Jun YW, Seo JW, Cheon A. Nanoscaling laws of magnetic nanoparticles and their applicabilities in biomedical sciences. Acc Chem Res. 2008; 41: 179-89.

44. Jun YW, Huh YM, Choi JS, Lee JH, Song HT, Kim S, Yoon S, Kim KS, Shin JS, Suh JS, Cheon J. Nanoscale size effect of magnetic nanocrystals and their utilization for cancer diagnosis via magnetic resonance imaging. J Am Chem Soc. 2005; 127: 5732-3.

45. Duan $H$, Kuang $M$, Wang $X$, Wang $Y A$, Mao $H$, Nie S. Reexamining the effects of particle size and surface chemistry on the magnetic properties of iron oxide nanocrystals: New insights into spin disorder and proton relaxivity. J Phys Chem C. 2008 ; 112: 8127-31.

46. Tromsdorf UI, Bigall NC, Kaul MG, Bruns OT, Nikolic MS, Mollwitz B, Sperling RA, Reimer R, Hohenberg H, Parak WJ, Forster S, Beisiegel U, Adam G, Weller H. Size and surface effects on the MRI relaxivity of manganese ferrite nanoparticle contrast agents. Nano Lett. 2007; 7: 2422-7.

47. Roch A, Gossuin Y, Muller RN, Gillis P. Superparamagnetic colloid suspensions: Water magnetic relaxation and clustering. J Magn Magn Mater. 2005; 293: 532-9.

48. Ai H, Flask C, Weinberg B, Shuai X, Pagel MD, Farrell D, Duerk J, Gao JM. Magnetite-loaded polymeric micelles as ultrasensitive magnetic resonance probes. Adv Mater. 2005; 17: 1949-52.

49. Berret JF, Schonbeck N, Gazeau F, El Kharrat D, Sandre O, Vacher A, Airiau M. Controlled clustering of superparamagnetic nanoparticles using block copolymers: Design of new contrast agents for magnetic resonance imaging. J Am Chem Soc. 2006; 128: 1755-61.

50. Chen H, Yeh J, Wang L, Khurshid H, Peng N, Wang AY, Mao $\mathrm{H}$. Preparation and control of the formation of single core and clustered nanoparticles for biomedical applications using a versatile amphiphilic diblock copolymer. Nano Res. 2010; 3 : 852-62.

51. Na HB, Lee JH, An K, Park YI, Park M, Lee IS, Nam DH, Kim ST, Kim SH, Kim SW, Lim KH, Kim KS, Kim SO, Hyeon T. Development of a $\mathrm{T} 1$ contrast agent for magnetic resonance imaging using $\mathrm{MnO}$ nanoparticles. Angew Chem Int Ed. 2007; 46: 5397-401.
52. Shin J, Anisur RM, Ko MK, Im GH, Lee JH, Lee IS. Hollow Manganese Oxide Nanoparticles as Multifunctional Agents for Magnetic Resonance Imaging and Drug Delivery. Angew Chem Int Ed. 2009; 48: 321-4.

53. Tong S, Hou S, Zheng Z, Zhou J, Bao G. Coating Optimization of Superparamagnetic Iron Oxide Nanoparticles for High T(2) Relaxivity. Nano Lett. 2010; 10: 4607-13.

54. Rohrer M, Bauer H, Mintorovitch J, Requardt M, Weinmann HJ. Comparison of magnetic properties of MRI contrast media solutions at different magnetic field strengths. Invest Radiol. 2005; 40: 715-24.

55. Lanza GM, Yu X, Winter PM, Abendschein DR, Karukstis KK, Scott MJ, Chinen LK, Fuhrhop RW, Scherrer DE, Wickline SA. Targeted antiproliferative drug delivery to vascular smooth muscle cells with a magnetic resonance imaging nanoparticle contrast agent implications for rational therapy of restenosis. Circulation. 2002; 106: 2842-7.

56. Smirnov $\mathrm{P}$, Lavergne E, Gazeau F, Lewin M, Boissonnas A, Doan B-T, Gillet B, Combadiere C, Combadiere B, Clement O. In vivo cellular imaging of lymphocyte tracking by MRI: A tumor model approach to cell-based anticancer therapy. Magn Reson Med. 2006; 56: 498-508.

57. Varallyay CG, Muldoon LL, Gahramanov S, Wu YJ, Goodman JA, Li X, Pike MM, Neuwelt EA. Dynamic MRI using iron oxide nanoparticles to assess early vascular effects of antiangiogenic versus corticosteroid treatment in a glioma model. J Cereb Blood F Met. 2009; 29: 853-60.

58. Bulte JWM, Brooks RA. Magnetic nanoparticles as contrast agents for MR imaging-An overview. In: Bulte JWM, Brooks RA, ed. Scientific and Clinical Applications of Magnetic Carriers, 1st ed. New York: Springer; 1997: 527-43.

59. Bulte JWM, Duncan ID, Frank JA. In vivo magnetic resonance tracking of magnetically labeled cells after transplantation. J Cereb Blood F Met. 2002; 22: 899-907.

60. Kraitchman DL, Heldman AW, Atalar E, Amado LC, Martin BJ, Pittenger MF, Hare JM, Bulte JWM. In vivo magnetic resonance imaging of mesenchymal stem cells in myocardial infarction. Circulation. 2003; 107: 2290-3.

61. Bulte JWM, Arbab AS, Douglas T, Frank JA. Preparation of magnetically labeled cells for cell tracking by magnetic resonance imaging. Method Enzymol. 2004; 386: 275-99.

62. Simon GH, Bauer J, Saborovski O, Fu YJ, Corot C, Wendland MF, Daldrup-Link HE. T1 and T2 relaxivity of intracellular and extracellular USPIO at $1.5 \mathrm{~T}$ and $3 \mathrm{~T}$ clinical MR scanning. Eur Radiol. 2006; 16: 738-45.

63. Korosoglou G, Weiss RG, Kedziorek DA, Walczak P, Gilson WD, Schaer M, Sosnovik DE, Kraitchman DL, Boston RC, Bulte JWM, Weissleder R, Stuber M. Noninvasive detection of macrophage-rich atherosclerotic plaque in hyperlipidemic rabbits using "positive contrast" magnetic resonance imaging. J Am Coll Cardiol. 2008; 52: 483-91.

64. Shapiro EM, Skrtic S, Koretsky AP. Sizing it up: Cellular MRI using micron-sized iron oxide particles. Magn Reson Med. 2005; 53: 329-38.

65. Cunningham $\mathrm{CH}$, Arai T, Yang PC, McConnell MV, Pauly JM, Conolly SM. POsitive contrast magnetic resonance imaging of cells labeled with magnetic nanoparticles. Magn Reson Med. 2005; 53: 999-1005.

66. Balchandani P, Yamada M, Pauly J, Yang P, Spielman D. Self-Refocused Spatial-Spectral Pulse for Positive Contrast Imaging of Cells Labeled with SPIO Nanoparticles. Magn Reson Med. 2009; 62: 183-92.

67. Stuber M, Gilson WD, Schaer M, Kedziorek DA, Hofmann LV, Shah S, Vonken EJ, Bulte JWM, Kraitchman DL. Positive contrast visualization of iron oxide-labeled stem cells using inversion-recovery with ON-Resonant water suppression (IRON). Magn Reson Med. 2007; 58: 1072-7. 
68. Dharmakumar R, Koktzoglou I, Li D. Generating positive contrast from off-resonant spins with steady-state free precession magnetic resonance imaging: theory and proof-of-principle experiments. Phys Med Biol. 2006; 51: 4201-15.

69. Zhang Z, Dharmakumar R, Mascheri N, Fan Z, Wu S, Li D. Comparison of Superparamagnetic and Ultrasmall Superparamagnetic Iron Oxide Cell Labeling for Tracking Green Fluorescent Protein Gene Marker with Negative and Positive Contrast Magnetic Resonance Imaging. Mol Imaging. 2009; 8: 148-55.

70. Zurkiya $\mathrm{O}, \mathrm{Hu} \mathrm{X}$. Off-resonance saturation as a means of generating contrast with superparamagnetic nanoparticles. Magn Reson Med. 2006; 56: 726-32.

71. Zhou R, Idiyatullin D, Moeller S, Corum C, Zhang H, Qiao H, Zhong J, Garwood M. SWIFT Detection of SPIO-Labeled Stem Cells Grafted in the Myocardium. Magn Reson Med. 2010; 63: 1154-61.

72. Small WC, Nelson RC, Bernardino ME. Dual Contrast Enhancement of Both T1-weighted and T2-weighted Sequences Using Ultrasmall Superparamagnetic Iron-oxide. Magn Reson Imaging. 1993; 11: 645-54.

73. Canet E, Revel D, Forrat R, Baldyporcher C, Delorgeril M, Sebbag L, Vallee JP, Didier D, Amiel M. Superparamagnetic Iron-oxide Particles and Positive Enhancement for Myocardial Perfusion Studies Assessed by Subsecond T1-weighted MRI. Magn Reson Imaging. 1993; 11: 1139-45.

74. Robson MD, Bydder GM. Clinical ultrashort echo time imaging of bone and other connective tissues. NMR Biomed. 2006; 19: 765-80.

75. Hogemann D, Ntziachristos V, Josephson L, Weissleder R. High throughput magnetic resonance imaging for evaluating targeted nanoparticle probes. Bioconjugate Chem. 2002; 13: 116-21.

76. Liu J, Cheng ECH, Long RC, Jr., Yang S-H, Wang L, Cheng P-H, Yang J, Wu D, Mao H, Chan AWS. Noninvasive Monitoring of Embryonic Stem Cells In Vivo with MRI Transgene Reporter. Tissue Eng. 2009; 15: 739-47.

77. Liu W, Dahnke H, Rahmer J, Jordan EK, Frank JA. Ultrashort T2* Relaxometry for Quantitation of Highly Concentrated Superparamagnetic Iron Oxide (SPIO) Nanoparticle Labeled Cells. Magn Reson Med. 2009; 61: 761-6.

78. Khemtong C, Togao O, Ren J, Kessinger CW, Takahashi M, Sherry AD, Gao J. Off-resonance saturation MRI of superparamagnetic nanoprobes: Theoretical models and experimental validations. J Magn Reson. 2011; 209: 53-60.

79. Langley J, Liu W, Jordan EK, Frank JA, Zhao Q. Quantification of SPIO Nanoparticles in vivo Using the Finite Perturber Method. Magn Reson Med. 2011; 65: 1461-9.

80. Zhao Q, Langley J, Lee S, Liu W. Positive contrast technique for the detection and quantification of superparamagnetic iron oxide nanoparticles in MRI. NMR Biomed. 2011; 24: 464-72. 